\title{
Bilateral carotid artery dissection in a SARS-CoV-2 infected patient: causality or coincidence?
}

\author{
Mauro Morassi $^{1}\left(\mathbb{D} \cdot\right.$ Barbara Bigni $^{2} \cdot$ Milena Cobelli $^{1} \cdot$ Lodoviga Giudice $^{3} \cdot$ Claudio Bnà $^{4} \cdot$ Alberto Vogrig $^{5}$
}

Received: 4 May 2020 / Revised: 5 June 2020 / Accepted: 8 June 2020 / Published online: 12 June 2020

c) Springer-Verlag GmbH Germany, part of Springer Nature 2020

\section{Dear Sirs,}

Evidence of cerebrovascular complications associated with SARS-CoV-2 is still limited [1] and the mechanisms of stroke in the context of Covid-19 remain incompletely understood. Herein we report the first case of Covid19-associated bilateral carotid artery dissection (CAD).

A 58-year-old man presented to the Emergency Department with fever and mild respiratory symptoms for 1 week associated with intense headache and neck pain (more intense on the left side, with anterior irradiation) which started 2 days before. Previous medical history was remarkable for low-grade oligodendroglioma treated with surgery. His treatment included levetiracetam and lacosamide for glioma-related epilepsy. Neurological evaluation revealed no abnormality. Chest CT showed bilateral ground-glass opacities and consolidations suggestive for Covid-19-related pneumonia (Fig. 1a). Nasopharyngeal swab was positive for SARS-CoV-2 on reverse-transcriptase-polymerasechain-reaction (RT-PCR) assay. Laboratory results showed increased C-reactive protein $(45.2 \mathrm{mg} / \mathrm{L}$, n.v. $<5)$, minimally increased lactate dehydrogenase (229 U/L, n.v.125-220) and

Mauro Morassi

mauro.morassi@poliambulanza.it

1 Department of Diagnostic Imaging, Unit of Neuroradiology, Fondazione Poliambulanza Istituto Ospedaliero, Via L. Bissolati 57, 25124 Brescia, Italy

2 Head and Neck Department, Unit of Neurology, Fondazione Poliambulanza Istituto Ospedaliero, Brescia, Italy

3 Head and Neck Department, Unit of Neurosurgery, Fondazione Poliambulanza Istituto Ospedaliero, Brescia, Italy

4 Department of Diagnostic Imaging, Unit of Radiology, Fondazione Poliambulanza Istituto Ospedaliero, Brescia, Italy

5 Clinical Neurology Unit, Azienda Sanitaria Universitaria Friuli Centrale, Presidio Ospedaliero, Santa Maria della Misericordia, Udine, Italy no evidence of respiratory distress $\left(\mathrm{SpO}_{2} 96 \%, \mathrm{PaO}_{2} / \mathrm{FiO}_{2}\right.$ 383). The patient's headache persisted during hospitalization, and it was only partially responsive to intravenous analgesics (ketorolac and paracetamol). On day 7, a brain CT-angiography showed a narrowed eccentric lumen of both internal carotid arteries, particularly on the left side, which started from the distal cervical part and extended towards the entrance into the petrous bone. MR-angiography confirmed the stenosis (Fig. 1b) and showed subacute mural hematomas (Fig. 1c-h) due to dissection. The patient did not manifest focal neurological deficits and no ischemic lesion was detected on diffusion-weighted brain MRI. On further questioning, the patient did no report history of heritable connective-tissue disorders and denied a recent head or neck trauma. Moreover, he did not present marfanoid habitus, highly stretchy skin, or joint hypermobility. He was initially treated with enoxaparin followed by warfarin. Interestingly, the retrospective evaluation of a brain MRI performed approximately 1 month before this admission (as part of low-grade glioma follow-up) revealed a normal "flow-void" signal on T2 images at the level of the terminal portion of extra-cranial internal carotid artery bilaterally, suggesting that arterial wall dissection occurred in close temporal association with the recent infection. At discharge (on day 22), the patient was still in good neurological conditions.

The temporal sequence in this case suggests, but does not prove, that Covid-19 represented a triggering factor for CAD. However, a body of epidemiological and clinical evidence suggest that infections, particularly those involving the respiratory tract, may represent a triggering factor for CAD [2-4]. Mechanical factors such as coughing, sneezing or vomiting could not account for this association [2, 3]. Interestingly, the association has been reported stronger for multiple rather than single artery dissection [4] as in the case here presented. Moreover, the absence of other predisposing factors in this patient suggests a potential role of SARS-CoV-2 as a trigger, even though a history of trauma is lacking in many cases of CAD $[3,4]$. 


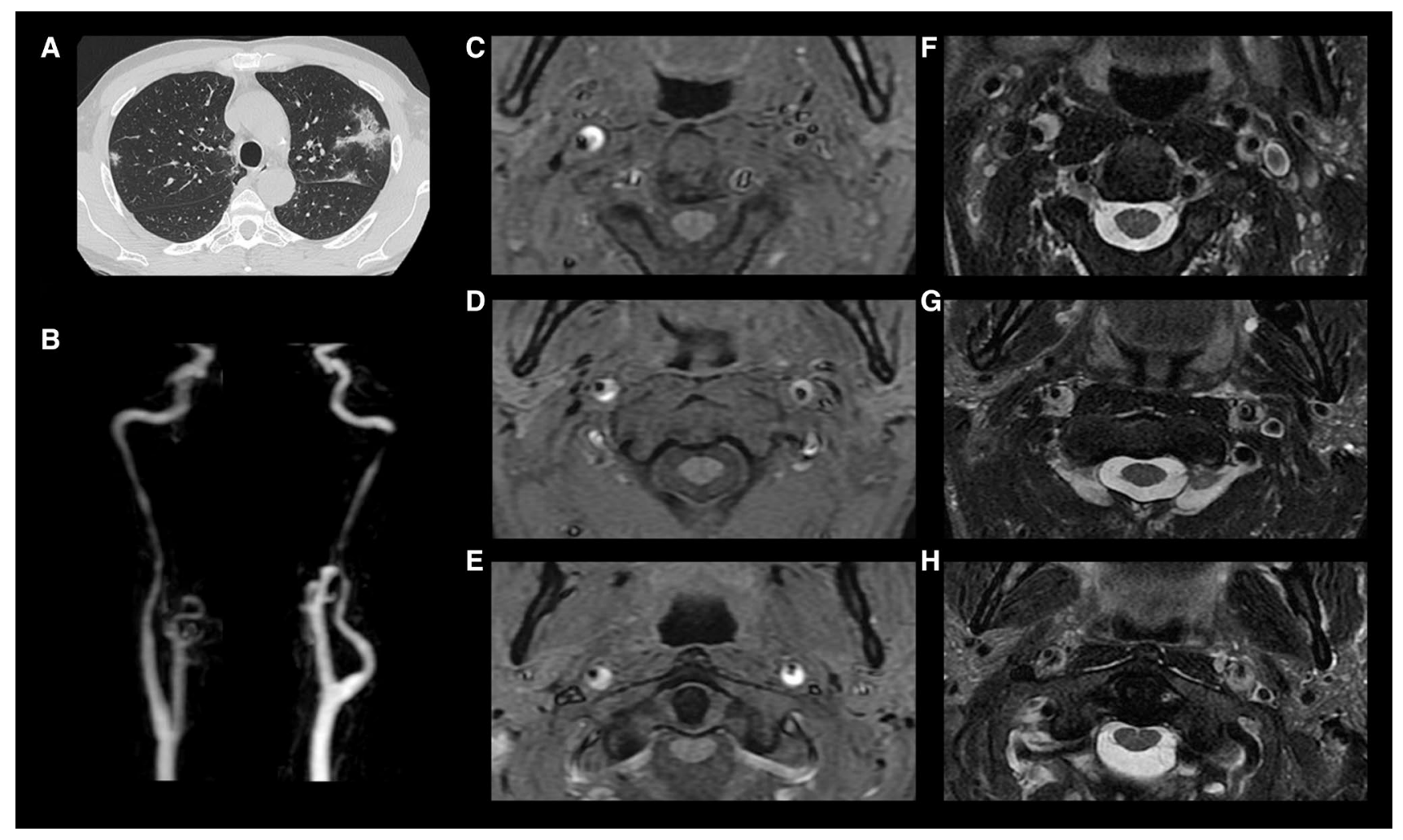

Fig. 1 Chest CT examination a showing focal ground glass opacities and consolidations in both lungs; bilateral severe stenosis of both carotid arteries on Maximum Intensity Projection reconstruction of contrast enhanced MR-angiography (b). Axial MRI images show a narrowed eccentric flow void surrounded by a crescent-shaped sig-

The alternative hypothesis of a coincidental association needs also to be considered. During a pandemic, it is possible that a large proportion of patients presenting with diseases pathogenically not related to the infection are actually infected, and this of course can apply to Covid19. Nevertheless, cerebrovascular complications of Covid19 are increasingly reported [5], and cases of dissection involving other arteries (including coronary artery [6] and aorta [7]) were recently observed, raising further concern. Interestingly, in the latter cases the patients were young (39- and 52-year-old, respectively) and healthy adults, without prior cardiovascular antecedents.

The mechanisms by which viral infections can trigger damage to artery walls may be direct or indirect. Recent evidence suggests that vascular dysfunction plays a major role in Covid-19 pathogenesis $[8,9]$. In particular, SARSCoV-2 may directly interact with endothelial cells by binding to ACE-2, which is highly expressed by vascular tissue, leading to endothelial activation, tissue damage and disordered cytokine release [8,9]. Alternatively, inflammatory cytokines (such as TNF- $\alpha$ and IL-1), may promote activation of matrix metalloproteinases and degradation of nal alteration of both ICA markedly hyperintense on T1-weighted FS images (c-e) and hyperintense on T2-weighted images (f-h) consistent with subacute mural hematomas. The lesions are showed at different levels: between $\mathrm{C} 2-\mathrm{C} 3(\mathbf{c}, \mathbf{f}), \mathrm{C} 2(\mathbf{d}, \mathbf{g})$ and at level of the anterior arch of $\mathrm{C} 1(\mathbf{e}, \mathbf{h})$

extra-cellular matrix [10], providing the conditions for a weaken vessel wall and CAD.

In conclusion, this case poses the question of a potential association between Covid-19 and CAD but more cases are necessary to support a causal relationship. Nevertheless, clinician should be aware of this possible association since a timely diagnosis of CAD can reduce the risk of stroke and long-term disability.

Funding The authors received no financial support for this work.

\section{Compliance with ethical standards}

Conflicts of interest None reported.

Ethical standard statement The patient provided written consent. All procedures were performed in accordance with the local ethics committee and the Declaration of Helsinki. 


\section{References}

1. Mao L, Jin H, Wang M et al (2020) Neurologic manifestations of hospitalized patients with coronavirus disease 2019 in Wuhan, China. JAMA Neurol. https://doi.org/10.1001/jamaneurol .2020.1127(published online April 10, 2020)

2. Grau AJ, Urbanek C, Palmet F (2010) Common infections and the risk of stroke. Nat Rev Neurol 6:681-694. https://doi.org/10.1038/ nrneurol.2010.163

3. Grau AJ, Brandt T, Buggle F et al (1999) Association of cervical artery dissection with recent infection. Arch Neurol 56(7):851856. https://doi.org/10.1001/archneur.56.7.851

4. Guillon B, Berthet K, Benslamia L et al (2003) Infection and the risk of spontaneous cervical artery dissection: a case-control study. Stroke 34(7):e79-e81. https://doi.org/10.1161/01. STR.0000078309.56307.5C

5. Morassi M, Bagatto D, Cobelli M et al (2020) Stroke in patients with SARS-CoV-2 infection: case series. J Neurol. https://doi. org/10.1007/s00415-020-09885-2(published online May 20)
6. Fernandez Gasso L, Maneiro Melon NM, Sarnago Cebada F et al (2020) Multivessel spontaneous coronary artery dissection presenting in a patient with severe acute SARS-CoV-2 respiratory infection. Eur Heart J. https://doi.org/10.1093/eurheartj/ehaa400

7. Fukuhara S, Rosati CM, El-Dalati S (2020) Acute type A aortic dissection during COVID-19 outbreak. Ann Thorac Surg. https:// doi.org/10.1016/j.athoracsur.2020.04.008

8. Leisman DE, Deutschman CS, Legrand M (2020) Facing COVID19 in the ICU: vascular dysfunction, thrombosis, and dysregulated inflammation. Intensive Care Med. https://doi.org/10.1007/s0013 4-020-06059-6(published online April 28, 2020)

9. Varga Z, Flammer AJ, Steiger P et al (2020) Endothelial cell infection and endotheliitis in Covid-19. Lancet. https://doi.org/10.1016/ S0140-6736(20)30937-5(published online April 17, 2020)

10. Lee HS, Noh JY, Shin OS et al (2020) Matrix metalloproteinase-13 in atherosclerotic plaque is increased by influenza A virus infection. J Infect Dis 221(2):256-266. https://doi.org/10.1093/ infdis/jiz580 